\title{
Effects on Alkaline Phosphatase Level in Crevicular Fluid Produced by Rapid Palatal Expansion with Hyrax Expander
}

\author{
Ahmad Shamim ${ }^{1}$ \\ Waheed-ul-Hamid ${ }^{2}$ \\ Muhammad Ilyas ${ }^{3}$ \\ Samina Qadir ${ }^{4}$ \\ Ushna Ahmad ${ }^{5}$
}

\author{
BDS \\ BDS, MS, MOrth, MCPS \\ BDS, FCPS \\ BDS, MDS \\ BDS
}

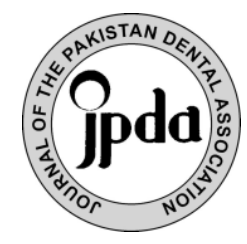

\begin{abstract}
:
Introduction: Rapid maxillary expansion (RME) is a dramatic and preferred procedure having a history of over 100 years. When a transverse maxillary Arch is diagnosed it is corrected by doing RME. Although several studies have been done in last four decades on gingival crevicular fluid, no data have been reported on the repeatability of gingival crevicular fluid collection and then following its quantification procedure. The objectives of this study were to examine the changes in alkaline phosphatase (ALP) activities in gingival crevicular fluid (GCF) during rapid palatal expansion and in retention period by hyrax, and to explore the relationship between these changes and periodontal tissue remodeling.

Methodology: Seventeen patients with age between 9-14 years were selected according to inclusion and exclusion criteria. Periodontal parameters, including probing depth, were recorded at 3 months after RME. Furthermore, the GCF ALP activity was measured at the buccal and palatal sites.

Results: Periodontal parameters were generally similar between the Testing and Control sites during the study, with the exception that probing depth underwent a slight increase at the Testing sites. It was observed that at baseline, the GCF ALP activity was similar between the buccal and Control sites; however, at 3 months of retention period a significantly greater enzymatic activity was seen at the Testing sites.

Conclusion: The results of our study concluded that in the retention phase of RME, there is reported increase in GCF ALP activity even at the end of 3 months of retention period with no clinically significant tissue inflammation. This increase in GCF ALP activity demands a longer period of retention phase may be up to 6 months of duration.

KEYWORDS: Gingival Crevicular Fluid, Alkaline Phosphatase, Rapid Palatal Expansion, Bone Remodeling, Hyrax Expander.

HOW TO CITE: Shamim A, Waheed-ul-Hamid, Ilyas M, Qadir S, Ahmad U. Effects on Alkaline Phosphatase Level in Crevicular Fluid Produced by Rapid Palatal Expansion with Hyrax Expander. J Pak Dent Assoc 2017; 26(2): 72-78.
\end{abstract}

Received: 27 April 2017, Accepted: 8 June 2017

\section{INTRODUCTION}

$\mathrm{R}$ apid maxillary expansion (RME) is a dramatic and preferred procedure having a history of over 100 years $^{1}$. The concept of orthopedic maxillary

1. Department of Orthodontics, de,Montmorency College of Dentistry, Lahore, Pakistan

2. Principal/ Head of Orthodontic Department, de,Montmorency College of Dentistry, Lahore

3-4. Assistant Professors, Department of Orthodontics, de,Montmorency College of Dentistry, Lahore, Pakistan

5. Clinical Observer, Department of Orthodontics, de,Montmorency College of Dentistry, Lahore, Pakistan

Corresponding Author: "Dr Ahmad Shamim"

<shamimahmad335@gmail.com> expansion was first described by Angell in a case report ${ }^{2}$. The normal trans palatal width of maxilla ranges from $35 \mathrm{~mm}$ to $39 \mathrm{~mm}$ and it suggests a bony palatal base of adequate size to accommodate a permanent dentition of average size $^{3}$. Expansion of the bony palatal base and width of dental arch allows us to have more space for the alignment of the crowded permanent dentition ${ }^{4}$. The expansion appliances commonly produces orthopedic forces in the range of 3 to 10 pounds ${ }^{5}$. Transverse maxillary constriction can be caused by various etiological factors including genetics, environmental and functional factors. The examples of different kind of maxillary expansion devices are Haas, Hyrax, Minnie expander, Cap Splints and recently developed acrylic 
bonded Rapid maxillary expander ${ }^{6}$. Expansion in skeletally mature patient can produce unwanted effects like lateral tipping of posterior teeth, extrusion of teeth, periodontal membrane compression, buccal root resorption, alveolar bone bending, fenestration of the buccal cortex, palatal tissue necrosis, inability to open the midpalatal suture, pain, and instability of the expansion. The factors that limit maxillary expansion in skeletally mature patients are numerous. These factors are related to changes with increasing age in the osseous articulations of the maxilla with the adjoining bones ${ }^{7}$. Gingival crevicular fluid (GCF) proves to be an exudate and the constituents present in it are derivative of microbial dental plaque, inflammatory cells present in it, host tissue, and serum. Different constituents present in the GCF gives information about diseases of periodontium and they have been proved as a diagnostic indicator of any damage to the tissues but few studies have paid attention on the aspect that the constituents of GCF give information about remodeling of bone also ${ }^{8,9}$. Alkaline phosphatase (ALP) is a hydrolase enzyme and its activity has been evident in bone-forming cells, and any change in the enzyme seen in serum and bone have been used as indicator for assessing metabolism of bone in a number of diseases. Acid and enzyme alkaline phosphatase in GCF of humans have been interrelated with the movement of teeth during the treatment of orthodontics and entire time period of appliance which is present in the mouth ${ }^{8,10-12}$. Higher alkaline phosphatase activities are associated with active bone formation ${ }^{13}$.

In orthodontics, exact timing of treatment plays an important role in the results of orthopedic treatment of dental and skeletal defects in growing patients. There are special maturational methods present which assess the skeletal maturity of the growing patients and they are based on radiographs like analysis of hand and wrist and the maturation of cervical vertebrae (CVM) method. These days other possibilities in the form of biochemical markers are obtainable and our patients can be benefitted from avoidance to unnecessary radiographic exposure. These agents are mainly involved in the growth of bone and its remodeling ${ }^{14}$. In GCF the first enzyme which was identified is ALP and it is essential for bone mineralization. In orthodontics the activity of GCF and ALP has been suggested as a diagnostic support $^{12,15,16}$.

Therefore this study is designed to examine the changes in activities of ALP in GCF during rapid palatal expansion and in retention period to explore the relationship between these changes and periodontal tissue remodeling. Hence aiding in correct timing for the treatment of skeletal problems as well as to reduce or increase the retention phase.

JPDA Vol. 26 No. 02 April-June 2017

\section{METHODOLOGY}

This study was conducted in the department of Orthodontics de'Montmorency college of Dentistry, Lahore and the ELISA of collected samples was done at University of Health Sciences, Lahore. The sample size was 17 patients and duration of study was 01 year. Patients were selected according to the Inclusion criteria. Patients with narrow maxilla that required palatal expansion, with good oral hygiene and periodontal status age between 9 to 14 years of either gender were selected. The exclusion criteria were Patients with compromised periodontal status, having any systemic disease like arthritis, diabetes, on antibiotic or NSAIDS therapy or those who refused to give consent. Patient's dental and medical history was obtained from patients and their parents before the commencement of study. Complete oral prophylaxis of the patients were made necessary with strict oral hygiene instructions and rinsing of mouth twice (twelve hourly) daily with 0.5 ounces of $0.2 \%$ chlorhexidine gluconate was started 1 week prior to the study and it was advised not to stop it as long the study continued. After taking the impression study cast was made and an appliance (bonded Hyrax expander) was fabricated and cemented in to the patient maxilla. One day before cementing appliance GCF was collected from the lingual and buccal sides of lower arch using paper strips and it was taken as control group. The gingiva was massaged to activate the GCF formation before the sample collection. The patient was advised to gargle strongly with a glass of sterile water to cleanse the oral cavity. The teeth was isolated by using a self-retaining retractor, use of suction and cotton rolls. The paper points were placed in the gingival crevice lingually and buccally to obtain GCF. In case of insufficient sample, the collection of GCF sample was repeated frequently like twice or three times until sufficient sample obtained. After the insertion of expander the expansion was done by activating the expander and it was .25 turn in the morning and .25 turn in the evening. GCF sample was taken with same technique after insertion of appliance on day 1, day 14, and day 21 and 3 months after active phase of expansion stopped subsequently. In the control group the collection of GCF was also taken on the same days. An upper occlusal x-ray was done after 1 week to confirm whether expansion has started or not. ALP level was measured in $\mathrm{mmol} / \mathrm{L}$ by ELISA technique.

\section{Statistical Analysis}

The collected data was entered and analyzed by using SPSS (Statistical Package for Social Sciences)version 20. The mean level of activity of enzyme alkaline phosphatase was calculated and the standard deviation of the mean values of the enzyme at the four sites was determined. Multivariant 
ANOVA analysis was applied to calculate any significance of enzyme activity among the predetermined 4 sites. A Pvalue $\leq 0.05$ was considered as statistical significant.

\section{RESULTS}

Out of 17 patients, $11(64.71 \%)$ were males and 6 $(35.29 \%)$ were females. The mean age of patient was $12.35 \pm 1.57$ and maximum number of patients was observed at age of 12 and 14 years. The descriptive statistics of alkaline phosphatase levels are shown in Table. A one-way analysis of variance (ANOVA) was used to analyze the mean of alkaline phosphatase levels among five groups (Day 0, Day 1, Day 14, Day 21 and Day 03 months). There was a statistically significant difference $(\mathrm{P}<0.05)$ in mean alkaline phosphatase levels in all five groups as shown in Table. The mean plot shows that mean alkaline phosphatase level increases from day 0 to day 90 (Fig. 1).

The assumption of homogeneity of variance was violated therefore Welch and Brown-Forsythe test were used ( $\mathrm{P}<$ 0.05) The mean difference of mean alkaline phosphatase level between day 1 and day 0 groups is $14.824 \pm 0.95$ with a significant difference of $\mathrm{P}<0.05$. The mean alkaline phosphatase level between day 14 and day 0 groups is $16.29 \pm 1.56$ with a significant difference of $\mathrm{P}<0.05$. The mean difference of mean alkaline phosphatase level between day 14 and day 1 groups is $1.471 \pm 1.50$ which is not significant $(\mathrm{P}>0.05)$.

A statistically significant difference $(\mathrm{P}<0.05)$ was observed between day 21 and day 0 , day 21 and day 1, day 21 and day 14 groups with mean difference of $24.47 \pm 1.65$, $9.64 \pm 1.59,8.17 \pm 2.01$ respectively. A statistically significant difference $(\mathrm{P}<0.05)$ was also observed between day 90 and day 0 , day 90 and day 1 , day 90 and day 14 , day 90 and day 21 groups with mean difference of $37.824 \pm 2.30$, $23 \pm 2.26,21.529 \pm 2.58,13.353 \pm 2.63$ respectively.

Post Hoc concludes that among five groups (day 0, day 1, day 14, day 21 and day 90), the mean alkaline phosphatase level are statistically significant in day 1 group vs day 0 group, day 14 group vs day 0 group, day 21 group vs day 0 group, day 21 vs day 1 , day 21 vs day 14 . A significant difference $(\mathrm{P}<0.05)$ was observed when day 90 was compared with day 0 , day 1 , day 14 and day 21 groups while no significant difference was seen between day 14 and day 1 groups $(\mathrm{P}>0.05)$.

Table 1. Descriptive Statistics of Alkaline Phosphatase Levels

\begin{tabular}{|c|c|c|c|c|c|c|c|}
\hline $\begin{array}{c}\text { Alkaline Phosphatase } \\
\text { Levels }\end{array}$ & N & Mean & Std. Deviation & Std. Error & Lower Bound & Upper Bound & Minimum \\
\hline \hline DAY 0 ALP & 17 & 10.29 & 3.057 & .741 & 8.72 & 11.87 & 5 \\
\hline DAY 1 ALP & 17 & 25.12 & 2.497 & .606 & 23.83 & 26.40 & 20 \\
\hline DAY 14 ALP & 17 & 26.59 & 5.691 & 1.380 & 23.66 & 29.51 & 18 \\
\hline DAY 21 ALP & 17 & 34.76 & 6.078 & 1.474 & 31.64 & 37.89 & 24 \\
\hline DAY 90 ALP & 17 & 48.12 & 9.006 & 2.184 & 43.49 & 52.75 & 31 \\
\hline Total & 85 & 28.98 & 13.688 & 1.485 & 26.02 & 31.93 & 51 \\
\hline
\end{tabular}

Table 2. One-Way Anova

\begin{tabular}{|c|c|c|c|c|c|}
\hline ALP_LEVELS & Sum of Squares & Df & Mean Square & F & Sig. \\
\hline \hline Between Groups & 13081.718 & 4 & 3270.429 & 98.498 & \\
\hline Within Groups & 2656.235 & 80 & 33.203 & & \\
\hline Total & 15737.953 & 84 & & \\
\hline
\end{tabular}




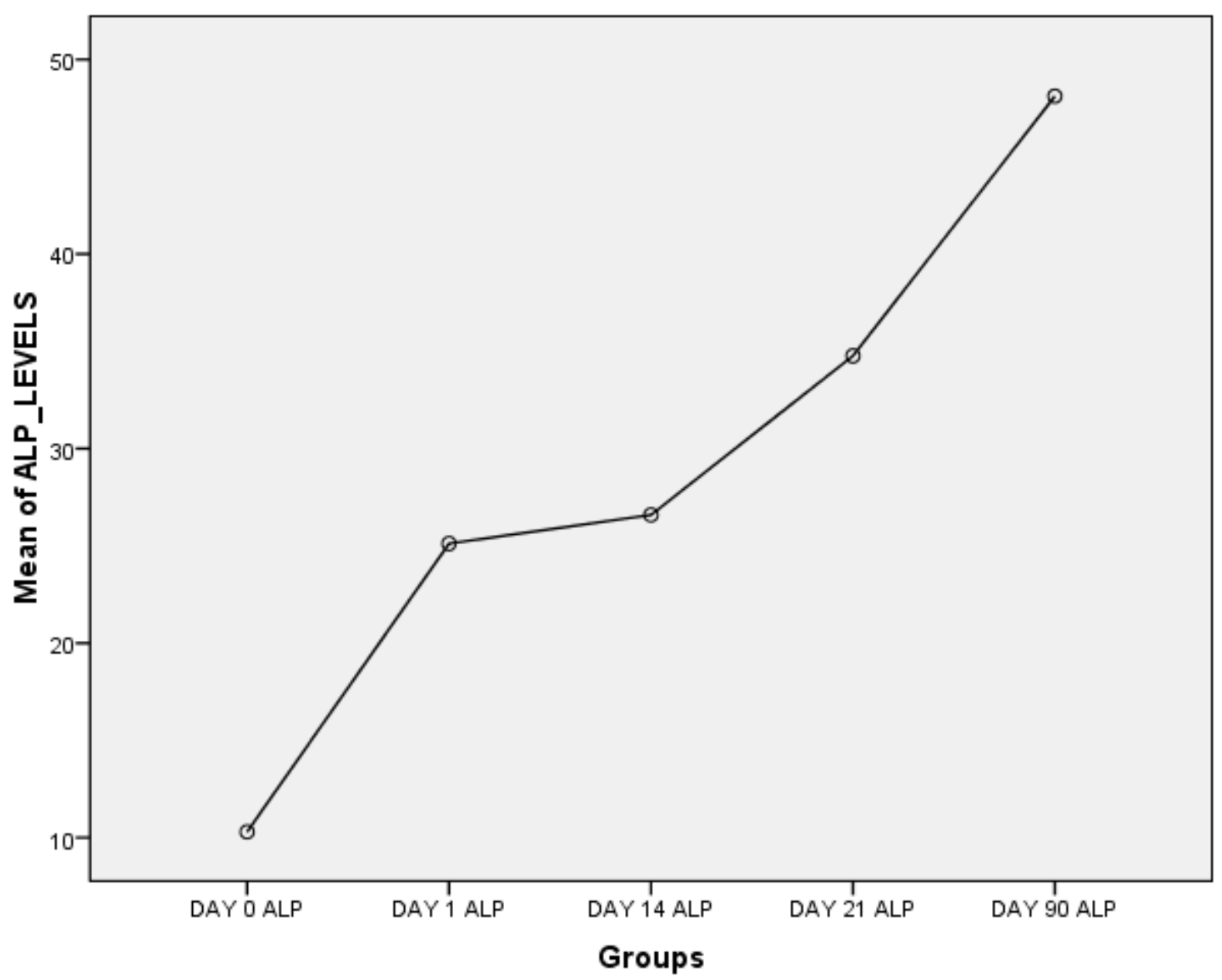

Fig. (1). Mean plot of alkaline phosphatase levels.

\section{DISCUSSION}

Constricted maxilla is one of the common reasons of the anomalous dentition. This anomaly may be due to different factors like genetics, environmental and functional factors $^{17,18}$.

It can be corrected by expansion of the palate and the most preferred method among growing patients is Rapid Maxillary Expansion ${ }^{6}$. The RME can be achieved with different methods which includes use of haas expander, Minnie expander, Cap splints, Niti expanders. In the study we used the bonded hyrax expander ${ }^{6}$.

Normally the RME is done in growing patients but if the subject is skeletally mature then expansion is done through surgically assisted $\mathrm{RME}^{19}$. The normal width of the maxilla is around $35 \mathrm{~mm}$ to $39 \mathrm{~mm}$ approximately which accommodates the dentition of average size $\mathrm{e}^{3,4}$. The patients which we selected for our study were evaluated and diagnosed with constricted maxilla before the commencement of the treatment. To assess the width of the maxilla we can use different methods like $\mathrm{x}$-rays and cast analysis but these days bio markers are providing new options and they are considered as diagnostic markers of different anomalies like bone diseases or periodontal diseases in mouth. The advantages of biomarkers are by checking their values we may keep our patients away from extra radiographic exposures, and they signify certain agents that are concerned mainly in bone growth and remodeling ${ }^{14}$.

Alkaline phosphatase is an enzyme which is easily detectable and it is used as a symbol of bone metabolism in numerous diseases ${ }^{8,12}$. As increased levels of ALP are associated with bone formation so these levels can play vital function in the onset of exact treatment timings of orthopedic intervention. Alteration of alkaline phosphatase 
Table 3. Multiple Comparisons Games-Howell

\begin{tabular}{|c|c|c|c|c|c|c|}
\hline \multirow[b]{2}{*}{ (I) Groups } & \multirow[b]{2}{*}{ (J) Groups } & \multirow[b]{2}{*}{ Mean Difference (I-J) } & \multirow[b]{2}{*}{ Std. Error } & \multirow[b]{2}{*}{ Sig. } & \multicolumn{2}{|c|}{ 95\% Confidence Interval } \\
\hline & & & & & Lower Bound & Upper Bound \\
\hline \multirow[t]{4}{*}{ DAY 0 ALP } & DAY 1 ALP & $-14.824^{*}$ & .957 & .000 & -17.60 & -12.05 \\
\hline & DAY 14 ALP & $-16.294^{*}$ & 1.567 & .000 & -20.90 & -11.69 \\
\hline & DAY 21 ALP & $-24.471^{*}$ & 1.650 & .000 & -29.34 & -19.60 \\
\hline & DAY 90 ALP & $-37.824^{*}$ & 2.307 & .000 & -44.74 & -30.91 \\
\hline \multirow[t]{4}{*}{ DAY 1 ALP } & DAY 0 ALP & $14.824^{*}$ & .957 & .000 & 12.05 & 17.60 \\
\hline & DAY 14 ALP & -1.471 & 1.507 & .863 & -5.94 & 3.00 \\
\hline & DAY 21 ALP & $-9.647^{*}$ & 1.594 & .000 & -14.39 & -4.90 \\
\hline & DAY 90 ALP & $-23.000^{*}$ & 2.267 & .000 & -29.84 & -16.16 \\
\hline \multirow[t]{4}{*}{ DAY 14 ALP } & DAY 0 ALP & $16.294^{*}$ & 1.567 & .000 & 11.69 & 20.90 \\
\hline & DAY 1 ALP & 1.471 & 1.507 & .863 & -3.00 & 5.94 \\
\hline & DAY 21 ALP & $-8.176^{*}$ & 2.019 & .003 & -14.01 & -2.34 \\
\hline & DAY 90 ALP & $-21.529^{*}$ & 2.584 & .000 & -29.08 & -13.98 \\
\hline \multirow[t]{4}{*}{ DAY 21 ALP } & DAY 0 ALP & $24.471^{*}$ & 1.650 & .000 & 19.60 & 29.34 \\
\hline & DAY 1 ALP & $9.647^{*}$ & 1.594 & .000 & 4.90 & 14.39 \\
\hline & DAY 14 ALP & $8.176^{*}$ & 2.019 & .003 & 2.34 & 14.01 \\
\hline & DAY 90 ALP & $-13.353^{*}$ & 2.635 & .000 & -21.03 & -5.68 \\
\hline \multirow[t]{4}{*}{ DAY 90 ALP } & DAY 0 ALP & $37.824^{*}$ & 2.307 & .000 & 30.91 & 44.74 \\
\hline & DAY 1 ALP & $23.000^{*}$ & 2.267 & .000 & 16.16 & 29.84 \\
\hline & DAY 14 ALP & $21.529^{*}$ & 2.584 & .000 & 13.98 & 29.08 \\
\hline & DAY 21 ALP & $13.353^{*}$ & 2.635 & .000 & 5.68 & 21.03 \\
\hline
\end{tabular}

*The mean difference is significant at the 0.05 level.

levels in gingival crevicular fluid is also an indicator of bone remodeling while orthodontic tooth movement ${ }^{12,20}$.

In the present study, the alkaline phosphatase levels were calculated at different times from zero hour to 21 days and then on 3 months after the active phase of expansion was over on day 21. At the experiment side, the alkaline phosphatase level reaches peak on the 14th day after placement/activation of orthodontic appliance. This finding matches with the earlier study ${ }^{21,22}$. It is detected that there is a decline in the level of alkaline phosphatase on the 21 st day in the experiment side which matches with the present study. This may be due to the reply of periodontium in response to the orthodontic force delivery over time ${ }^{20}$.

There has been a single study available on humans which checked the action of ALP and GCF related with RME treatment and it did not mainly checked the tension sites and it mentioned a time period of only 28 days follow up, but showed the bone turnover markedly longer ${ }^{21}$. The ALP is a enzyme which is involved mainly in bone mineralization through the process of hydrolyzing inorganic 
pyrophosphate $^{23}$, and the rate of bone formation is widely associated with $\mathrm{it}^{24}$. Alkaline phosphatase is an activity of bone formation cells i.e. Osteoblasts.

Alteration of alkaline phosphatase levels in gingival crevicular fluid is also an indicator of bone remodeling while orthodontic tooth movement ${ }^{20,12}$. In the present study, the alkaline phosphatase levels were calculated at different times from zero hour to 21 days. At the experiment side, the alkaline phosphatase level reaches peak on the 14th day after placement/activation of orthodontic appliance. This finding matches with the previous study ${ }^{21,22}$. Batra and colleagues also detected a decline in the level of alkaline phosphatase on the 21st day in the experiment side which matches with the present study ${ }^{20}$. This may be due to the reply of periodontium in response to the orthodontic force delivery over time. Keeling and colleagues in a study, showed that alkaline phosphatase is also an indicator of bone remodeling while orthodontic tooth movement ${ }^{25}$.

It is important to mention here that the results showed that GCF and ALP activity noted at the end of 3 months of retention period was still raised as we compare it with the 21 days of activation which showed a decline in their level. This raised level has suggested us that we should increase the retention period to more than 3 months may be up to 6 months after the removal of appliance. The results indicated that the formation of alveolar bone is still active even after the retention phase was over. This suggested that these results are comparable with other studies which have been reported earlier and even though with RME we obtain mainly the skeletal effects especially in the prepubertal phase of growth, but still we obtain dentoalveolar effects too ${ }^{26}$.

Although other studies reported that when the orthodontic treatment commences the chances of increased gingival swelling and plaque build up is a normal phenomenon $^{27}$, so it may be a cause of increased ALP level but good hygiene of oral cavity measures during the treatment of orthodontics can decrease in plaque buildup and swelling of the gums ${ }^{28}$. Moreover, we can avoid major biases by doing some split mouth protocol studies that effect the GCF and ALP levels during some systemic and local metabolic conditions.

The clinical significance of the results of our study shows that even at 3 months post RME the formation of alveolar bone at the tension sites of the teeth which are supported is still alive which needs to extend retention period up to 6 months so that proper bone formation and mineralization of these sites takes place with very less chances of relapse. As after the 3 months of retention period chances of relapse are very much high even after the use of retention appliance (Transpalatal arch with extended arms) and for patients it is not feasible to wear the appliance for the second time after the relapse of expansion.

Recently a study has been done by some researchers who recommended that as the bone formation and mineralization actively goes on even at 6 months so the retention period after the expansion of maxilla may be increased from 3 months to 6 months for proper formation of bone to avoid relapse $^{29}$. In our study, we checked the levels of GCF and ALP till 3 months after RME to assess formation of alveolar bone at the pressure sites of teeth which are supported. As at base line the GCF and ALP activity was alike at both testing and control sites but as the expansion started it raised significantly especially at day 14 . This activity decreased around day 21 but the results showed that GCF and ALP levels were significantly increased even at the end of 3 months of retention period and no marked tissue inflammation was seen. This enhances the support for an increase in retention period may be up to 6 months so that a very negligible chance of relapse may be noted.

\section{CONCLUSIONS}

The results of our study concluded that in the retention phase of RME, there is detection of increase in GCF ALP activity even at the end of 3 months of retention period with no clinically significant tissue inflammation. This increase in GCF ALP activity demands a longer period of retention phase may be up to 6 months of duration.

\section{REFERENCES}

1. Lamparski, DG, Rinchuse DJ, Close JM. Comparison of skeletal and dental changes between 2 point and 4 point rapid palatal expanders. Am J Orthod Dento fac Orthop 2003; 123: 321-28.

2. Angell EH. Treatment of irregularity of the permanent or adult teeth. Dent Cosmos 1860; 1: 540-44, 599-600.

3. Saeed MQ, Naeem S, Zaigham AM. A Comparison of the effects of rapid maxillary expansion screw height (deep vsshallow ) on the dentoalveolar structures in maxillary constricted patients. Biomedica 2004; 20: 9095.

4. Saeed MQ, Naeem S, Zaigham AM. A Comparison of the effects of rapid maxillary expansion screw height (deep vsshallow ) on the dentoalveolar structures in maxillary constricted patients. Biomedica 2004; 20: 9095.

5. Saeed MQ, Qazi H, Ahmad F, Zaigham AM. Effects of anterior maxillary expansion. A postero-anterior cephalometric evaluation J Pak Dent Assoc. 2009; 18: 102-6. 
6. Wendling LK, James A, McNamara JA, Franchi, L, Baccetti, T. Prospective study of the short-term treatment effects of the acrylic-splint rapid maxillary expander combined with the lower Schwarz appliance. Angle Orthod, 2004; 75: 7-14.

7. Suria L, Tanejab P. Surgically assisted rapid palatal expansion: A literature review. Am J Orthod Dentofacial Orthop 2008; 133: 290-302

8. Perinetti G, Paolantonio M., Attilio MD, Archivio DD, Tripodi D, Femminella B, Festa F, Spoto G. Alkaline phosphatase activity in gingival crevicular fluid during human orthodontic tooth movement. Am J Orthod Dentofacial Orthop 2002; 122: 548-56

9. Lamster IB. The host response in gingival crevicular fluid: potential applications in periodontitis clinical trials. J Periodontol 1992; 63: 1117-23.

10. Delmas PD. Clinical use of biochemical markers of bone remodeling in osteoporosis. Bone 1992; 13: 17-21.

11. Farley JR., Hall SL, Ritchie C, Herring S, Orcutt C, Miller BE. Quantification of skeletal alkaline phosphatase isoenzyme in canine serum. J Bone Miner Res 1992; 7: 779-92.

12. Insoft M, King GJ, Keeling SD. The measurement of acid and alkaline phosphatase in gingival crevicular fluid during orthodontic tooth movement. Am J Orthod Dentofacial Orthop 1996; 109: 287-96

13. Mishina Y, Starbuck MW, Gentile MA, Fukuda T, Kasparcova V, Seedor JG, Hanks MC, Amling M, Pinero GJ, Harada SI, Behringer RR. Bone morphogenetic protein type IA receptor signaling regulates postnatal osteoblast function and bone remodeling. J Biol Chem 2009; 279: 27560-66.

14. Perinetti G, Baccetti, T, Contardo L, Di Lenarda R. Gingival crevicular fluid alkaline phosphatase activity as a non-invasive biomarker of skeletal maturation. Orthod craniofac res 2001; 14: 44-50.

15. Rodan GA. Introduction to bone biology. Bone 1992; 13: S3-S6.

16. Perinetti G, Paolantonio M, D'Attilio M, D'Archivio D, Tripodi D, Femminella B, Festa F, Spoto G. Alkaline phosphatase activity in gingival crevicular fluid during human orthodontic tooth movement. Am J Orthod Dentofac Orthop 2002; 122: 548-56.

17. Duruk C, Sokucu O, Sezer H, Canbaye E. Evaluation of nasal airway resistance during rapid maxillary expansion using acoustic rhinometry. Eur J Orthod 2004; 26: 397-401.
18. Ozbek M, Memikoglu T, Altug-Atac A, Lowe A. Stability of maxillary expansion and tongue posture. Angle Orthod. 2009; 79: 214-9.

19. Haas AJ. The treatment of maxillary deficiency by opening the midpalatal suture. Angle orthod 1965; 35: 200-17.

20. Batra P, Kharbanda OP, Duggal R, Singh N, Parkash H. Alkaline phosphatase activity in gingival crevicular fluid during canine retraction. Orthod Craniofac Res 2006; 9: 44-51.

21. Wei FL, Wang CL, Liu DX, Guo J, Ke HF, Guo XX. Changes of aspartate aminotransferase and alkaline phosphatase activities in gingival crevicular fluid during rapid palatal expansion. Shanghai kou qiang yi xue= Shanghai J stomatol 2007; 16: 168-71.

22. D'Attilio M, Filippi MR, Femminella B, Festa F, Tecco S. The influence of an experimentally-induced malocclusion on vertebral alignment in rats: a controlled pilot study. Cranio 2005; 23: 119-29.

23. Robison R Soames KM. The possible significance of hexosephosphoric esters in ossification: Part II. The phosphoric esterase of ossifying cartilage. Biochem J 1924; 18: 740.

24. Christenson RH. Biochemical markers of bone metabolism: an overview. Clin biochem 1997; 30: 57393.

25. Keeling SD, King GJ, McCoy EA, Valdez M. Serum and alveolar bone phosphatase changes reflect bone turnover during orthodontic tooth movement. Am J Orthod Dentofac Orthop. 1993; 103: 320-26.

26. Lagravere MO, Major PW, Flores-Mir C. Long-term skeletal changes with rapid maxillary expansion: a systematic review. Angle orthod. 2005; 75: 1046-52.

27. Trossello VK, Gianelly AA. Orthodontic treatment and periodontal status. J periodontal. 1979; 50: 665-671.

28. Lundström F, Hamp SE, Nyman S. Systematic plaque control in children undergoing long-term orthodontic treatment. Euro J Orthod. 1980; 2: 27-39.

29. . Perinetti G, D’Apuzzo F, Contardo L, Primozic J, Rupel K, Perillo L. Gingival crevicular fluid alkaline phosphate activity during the retention phase of maxillary expansion in prepubertal subjects. A splitmouth longitudinal study. Am J Orthod Dentofac Orthop. 2015; 148: 90-96. 